\title{
END TO END QUALITY OF SERVICE ASSURANCE FOR MULTI-SERVICE PROVISIONING IN MOBILE AD HOC NETWORKS
}

\author{
Prabesh Dongol ${ }^{1}$, Dhadesugoor R. Vaman ${ }^{2}$ \\ 1 - PhD student, Department of Electrical and Computer Engineering, Prairie View \\ A\&M University, Prairie View, Texas, USA \\ 2 - Texas A\&M Regents Professor, TI Endowed Chair, and ARO CeBCom Director, \\ ECE Department, Prairie View A\&M University, Prairie View, Texas, USA
}

\begin{abstract}
Multimedia streaming over Mobile Ad Hoc networks has been a very challenging issue due to the dynamic behavior and uncertain nature of the channels. Transmission of real time video has bandwidth, delay and loss requirements. However there are no Quality of Service $(Q o S)$ guarantees for video transmission in today's network. There are many challenging issues that need to be addressed in designing mechanisms for video transmission, which include end-to-end Quality of Service, Bandwidth, Delay, Loss, Congestion, and Heterogeneity. The Challenges of delivering Multi-media signals are even pronounced in Wireless Networks (Mobile Ad Hoc Networks, Wireless Fidelity (Wi-Fi) and Cellular Networks) which are heavily bandwidth constrained and have no fixed infrastructures. In this Research we provide a theoretical model for minimum buffer size as a means of achieving smoother, higher quality streaming video. This Research presents a general optimal video smoothing algorithm based on the concept of dynamically controlled Coefficient of Variance $(\mathrm{CV})$, which is the ratio of standard deviation of the end-to-end delay and the expected value of the delay for each ensemble of packets being transmitted through the network. The results discuss how the size of the "receive buffer" is affected by the allocated bandwidth for each source-pair end users for supporting video streaming applications without any gaps. The simulation performance show that the dynamic client buffer size based on measured bandwidth variation achieves negligible jitter in the video streaming which is subjectively acceptable.
\end{abstract}

\section{KEYWORDS}

Quality of Service, Coefficient of Variance, Mobile Ad Hoc Networks

\section{INTRODUCTION}

Video streaming and video conferencing applications are becoming very important as internet is finding explosive opportunity to support a wide variety of hand-held devices, desktops and laptops. Service providers are attempting to increase bandwidth efficiency and minimization of service disruptions. End user devices want high bandwidth and are driving spectrum efficiency. Service provisioning is occurring in both wired and wireless networks and the QoS assurance need to be maintained if service providers have to support differentiated services for economic benefit. Transmission of real time video has bandwidth, delay and loss requirements. However there is no quality of service (QoS) guarantee for video transmission over the current internet. There are many challenging issues that need to be addressed in designing mechanisms for Internet 
International Journal of Network Security \& Its Applications (IJNSA), Vol.6, No.4, July 2014

video transmission. The challenging QoS issues are Bandwidth, Delay, Loss, Congestion, and Heterogeneity [1].

Quality of service (QoS) in the context of provisioning of network centric multi-services that includes transport of voice, video and data either homogenously or in mixed mode requires support of bounded information rate, bounded response time and bounded information loss. The quantification if these three parameters that are desirable is a function if the end user application. QoS assurance on the other hand requires maintaining the network connectivity at all times between two end users irrespective of what happens to the network path in terms of path failure or mobility while maintaining the desired QoS end to end.

In order to maintain end to end assurance there need to support cooperative dynamic control of information flow at the IP protocol layer for all multi service applications for each source destination pair. This needs to be achieved without any request for control information from the network. This is part of "user control" where the control information is exchanged between the source and destination using management packet between two remote agents along with an ensemble of IP packets that carry user application data. The network provider can further improve the ability to support end to end QoS provisioning by optimizing the multi hop path by maintaining high probability of path continuity for each source destination pair. This needs to be achieved in the presence of mobility, multi path fading and Doppler effects in wireless channels and in the presence of congestion in wired channels. The transport QoS is controlled by the network provider while the application QoS is controlled by the source destination pair.

QoS issues in current Internet has survived by increasing physical layer link capacity to meet its demand. Usually, IP layer assumes that a LAN rarely drops and delays packets. However, in WLANs due to the challenges of wireless channel, it makes Physical layer data rate improvements difficult to achieve. This is because error rate can be more than three orders of magnitude larger in WLANs than that of wired LAN. Furthermore conventional wireless networks are often connected to wired network and require a fixed wired backbone infrastructure. There is another type of wireless network that has neither a fixed base station nor has a wired backbone infrastructure. These kind of networks are called Mobile Ad Hoc Networks (MANET) that consists of mobile nodes acting as both mobile hosts and mobile routers. A wireless ad hoc network can be defined as an autonomous system of mobile nodes that are free to move randomly and organize themselves arbitrarily. Being self-organized and operating without existing infrastructures, MANET have several unique features. First their topology are dynamic due to mobility of nodes. Due to mobility of nodes, the topology of the network may change frequently. Thus node connectivity and link properties such as capacity and bit error rate cannot be predetermined. Next, the transmission medium is bandwidth constrained and time varying. Other factors such as path loss, fading, channel error and interference reduces capacity of wireless link. Finally wireless ad hoc networks are also power constrained that reduces transmission rate, data rate and processing speed of devices. These mobile ad hoc networks are expected to play an important role in commercial and military usage.

Given the features above, multimedia streaming over wireless MANET is a challenging task. Multimedia applications have bandwidth, delay and loss requirements. Therefore providing quality of service (QoS) support in the network is a challenging task. Amid difficulty, providing QoS in the network is unavoidable because of increasing popularity of multimedia applications and potential commercial usage of MANET. A reliable streaming mechanism is therefore required to guarantee that these applications are completely received at the destination.

In this paper, we try to focus on transmission of real time video, like live event or video conference, in MANET network environment and find a minimum buffer at the receiver node while maintaining video QoS. We use queuing theory analysis to provide QoS measures: Average 
International Journal of Network Security \& Its Applications (IJNSA), Vol.6, No.4, July 2014

delay, Variation in delay, Loss ratio and Delay statistics.

\section{BACKGROUND WORK}

Previous research has developed extensive work on delivering end to end QoS and QoS assurance for Voice/IP applications. Provisioning of Voice/IP service in Internet is not a challenge but achieving QoS and QoS assurance in the network is centric using IP as the seamless transport protocol is a significant challenge, since IP is a connectionless service and has no error correction support or bandwidth control within the peer to peer operation. Also the network providers have no control on the access networks and end user devices and their performance abilities. Thus achieving QoS and QoS assurance by the network providers is very difficult [2].

A novel technique of dynamic tri mode control of IP packets that allows high quality assurance of Voice/IP service provisioning for each source destination pair with cooperative management control. Dynamic controls are exercised based on Coefficient of Variance (ratio of standard deviation of delay and expected value) that varies when network path is congested or operating under normal conditions with reasonable traffic. The Coefficient of Variance refers to a statistical measure of the distribution of data points around the mean. The Coefficient of variance is a helpful statistic in comparing the degree of variation from one data series to the other although the means are considerably different from each other. Distributions with a coefficient of variance to be less than 1 are considered to be low variance whereas those with a coefficient of variance higher than 1 are considered to be high variance. It has been demonstrated both theoretically and by real implementation; tri mode control has effectively assured high probability of QoS for Voice/IP application end to end. The subjective quality of Voice is maintained at nearly toll quality for all conditions the network [2]. There are three modes of control considered: When Coefficient of Variance $(\mathrm{CV})$ is between 0.27 and 0.33 , the channel is considered to be in "normal mode" as such no control is necessary. When CV exceeds 0.33 , the standard deviation increases while maintaining normal expected value. In this case, the channel experience packet queuing in the hops across the connected path. The intent is to reduce the traffic from source. The packet delay in the ensemble is increased by one packet generation time and therefore indirectly reduce the offered traffic from source. This will reduce CV and the mode is referred to as "extended buffer" mode. The channel remains in extended buffer mode until the CV becomes less than 0.27 , thus hysteresis has been built to allow for smooth change of control without any oscillations in the modes. When CV is less than 0.27 , a condition occurs on the channel where congestion occurs in the path and packets get queued for a longer time and packets can be lost. The standard deviation is very low. In this mode, which is referred to as "sub-packet mode", the intent is to decrease the expected value of the delay to increase $\mathrm{CV}$ to be more than 0.27 to move it back to normal mode. The packets are split into "odd samples" and "even samples" packets and only "odd samples are transmitted. The even samples are interpolated at the receiver. This will increase the expected delay and move the channel to normal mode. The number of packets where we only send odd samples can be restricted to maintain higher QoS. If all packets are transmitted with only odd samples, there is a small degradation in the quality. 
The receiver buffer plays a major role in overall performance of streaming video. The required size of this buffer is affected by mainly by two conditions: encoding rate of the video and the quality of network. Since the buffer stores the next video packets to read for content player, we never want the player to reach a state where the buffer is empty. Filling the buffer takes time as the upcoming bytes over the network needs to be downloaded. In order to deliver a satisfying viewer experience the player buffer behavior needs to balance the initial load time against the risk that the buffer becomes empty leading to video that pauses or plays in stops and starts [3].

It has been pointed out by many researchers [4], [5], [6], [7] that video applications should be fair to other applications that have comparatively low data dates. This is often phrased as "TCP Friendliness" [4]. This has led to the development of adaptive video streaming applications that feature rate control in response to changing network conditions.

Transmission rate should be adjusted such that the buffer will never overflow or underflow. The buffer underflows when a frame fails to arrive before its playout time and the buffer overflows when the fill rate is higher than its drain rate. So to avoid underflow and overflow, the sequence of transmission rates must satisfy [5],

$$
\sum_{i=1}^{k} s_{i}-B_{\text {init }} \leq \sum_{i=1}^{k} r_{i} \leq \sum_{i=1}^{k} s_{i}-B_{\text {init }}+B_{\text {client }}
$$

Where $s_{i}$ (bits) is size of a frame, $B_{\text {client }}$ is the size of client buffer and $B_{\text {init }}$ (bits) is the amount of buffer filled initially. In this research, we propose to develop extensions to tri mode control to video streaming application where $\mathrm{CV}$ of each ensemble can be used to quantize receiver buffer size in such a way that performance can meet real time continuous video stream.

\section{SYSTEM ARCHITECTURE}

Multimedia streaming in Ad Hoc Network is a challenging issue due to dynamic behavior and uncertain nature of channels. In Ad Hoc Network it is not practical to assign large buffer size for the devices that have lower memory for buffering. However, receiver buffer plays a crucial role in overall performance of multimedia streaming. In video streaming application, digital video is transported across the network and the destination stores some portion of video prior to playing. The amount of storage should be optimized such that the end user witnesses continuous video transmission. In spite of technological evolutions in video compression, video streaming still requires large storage at the receiver side. So our objective is to design a buffering strategy with lower demands on buffer space while maintaining video QoS.

In a typical streaming session, the client requests video from a video server. For a smooth playout, video streaming needs a steady rate but because of congestions and packet loss in the network path, the transmission rate will decrease causing bandwidth variation which can cause degradation in video quality. Video conferencing on the other hand allows multiple windows to be set up on the screen and the video with associated data and voice are bundled to ensure the window to maintain lip sync and continuous motion of video approximately. There is limited storage in the conferencing application. Therefore a buffer can be placed in the client end where video frames are temporarily stored in order to smooth out bandwidth variations In order to use IP transport, it is critical to determine a basis of normalizing the storage at the receiving side as a function of sustainable data rate on the path. 
Storage Index, $S I=\left[\frac{\left(\frac{B}{T_{e}}\right)}{R_{e}}\right] \quad 0 \leq S I \leq 1$

Figure 1 below shows the role of client buffer in video streaming.

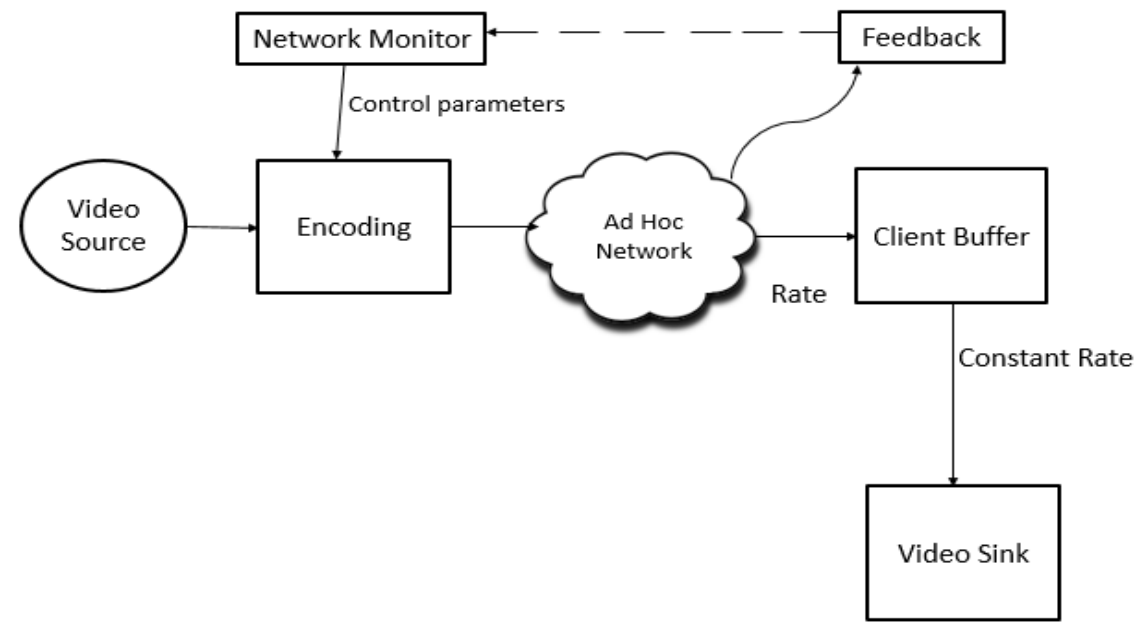

Figure 1: System Architecture

There are three mechanisms for congestion control namely; Rate control, rate adaptive video encoding and rate shaping [9]. Rate control follows the transport approach. Rate adaptive video encoding follows the compression approach and the rate shaping could follow either the transport approach or compression approach. Under our architecture, we adapt a Source Based Rate Control System that can minimize congestion by matching the rate of video stream to the available network bandwidth. The sender adapts the transmission rate of the video stream. A QoS monitor is maintained at the receiver side which measures behavior of arriving packets like packet loss and delay. Based on this feedback information about the network, the sender regulates the rate of video stream. Thus source based rate control can minimize the amount of packet loss by matching the rate of video stream to the available network bandwidth.

In this Research, we first intend to find minimum buffer size as a means of achieving smoother, higher quality streaming video. In the second case we are interested in smoothing the transmission rate by dynamically controlling the Coefficient of Variance, considering the buffer and delay constraints. We simulate a video playout to determine the number of pauses and the minimum buffer size for no interrupts to occur based on bandwidth variations. Once filled, the buffer is drained at the encoding rate of the video and whenever empty, number of interrupts is counted. By increasing the buffer size and repeating the simulation, we determine the smallest buffer size when there are no more interrupts. We assume that transmission and play out take place in time slots of constant length. We use relationship between the coefficient of variation of the trace and minimum buffer size and predict the perfect buffer size from the sample of the whole trace. 
International Journal of Network Security \& Its Applications (IJNSA), Vol.6, No.4, July 2014

\subsection{Buffer Design for Video Streaming Traffic in Ad Hoc Network}

Receiver buffer plays an important role in overall performance of streaming video. However in MANET it is not practical to allow large buffer sizes for the devices that have limited memory for buffering. The characteristics of traffic model is extremely important for buffer designing. An accurate traffic model of video sequence is necessary to predict network performance like end to end delay and packet loss. The most common way to use video traffic model in network is to perform live experiment on real network and real sources. However, testing on real networks can be expensive and cannot be used in a queuing models. An alternative to this kind of modeling is using mathematical analysis. Various stochastic video traffic models have been proposed in literature like Poisson based, Markov process based, wavelet based, self-similar and fractional Brownian based [16]. Statistical and mathematical traffic model can be better used in understanding various traffic characteristics. The Poisson process has been traditionally used for modeling voice and data processes. Markov modulated video traffic have been very popular for modeling different types of videos and with different compression schemes.

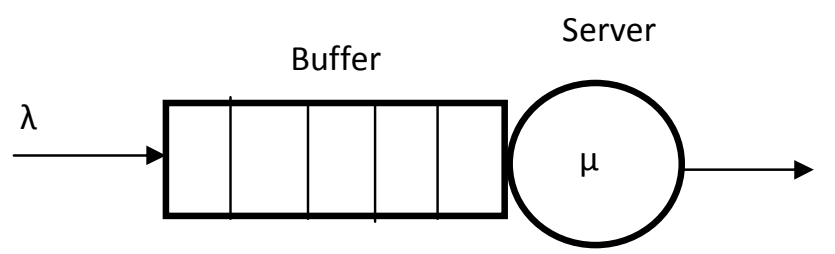

Figure 2. A simple queue system

We consider a Classical Markov Queuing Theory for M/M/1/B queue where packet arrival follows Poisson process with arrival rate $\lambda$ packets/second, packet service time is exponentially distributed with rate $\mu$ packets/second and a buffer size of B packets. Then, $\rho=\frac{\mu}{\lambda}$ is link load.
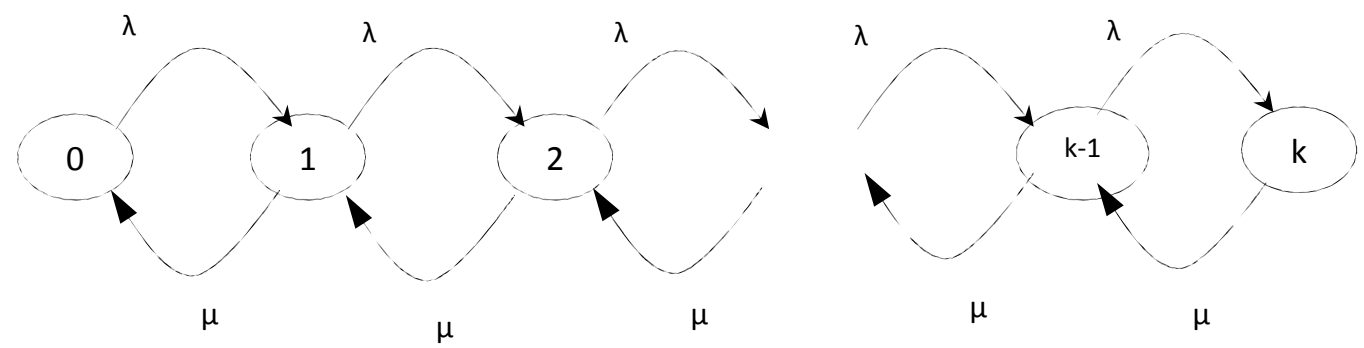

Figure 3. Birth death process state transition rate diagram

The Buffer Overflow Probability for such queue is,

$$
P_{\text {overflow }}=\frac{1-\rho}{1-\rho^{B+1}} \rho^{B}
$$

Solving equation 1 , we can find the closed form solution for minimum buffer capacity B to yield a desired overflow probability.

$$
B=\frac{\ln \left(P_{\text {overflow }}\right)}{\ln (\rho)}
$$


International Journal of Network Security \& Its Applications (IJNSA), Vol.6, No.4, July 2014

Based on this equation we can compare the minimum buffer required to meet a particular overflow objective for a Markovian queuing system. A figure for required buffer size as a function of offered load is shown in figure 3 for various overflow probability. We see that buffer size increases as the offered load increases.

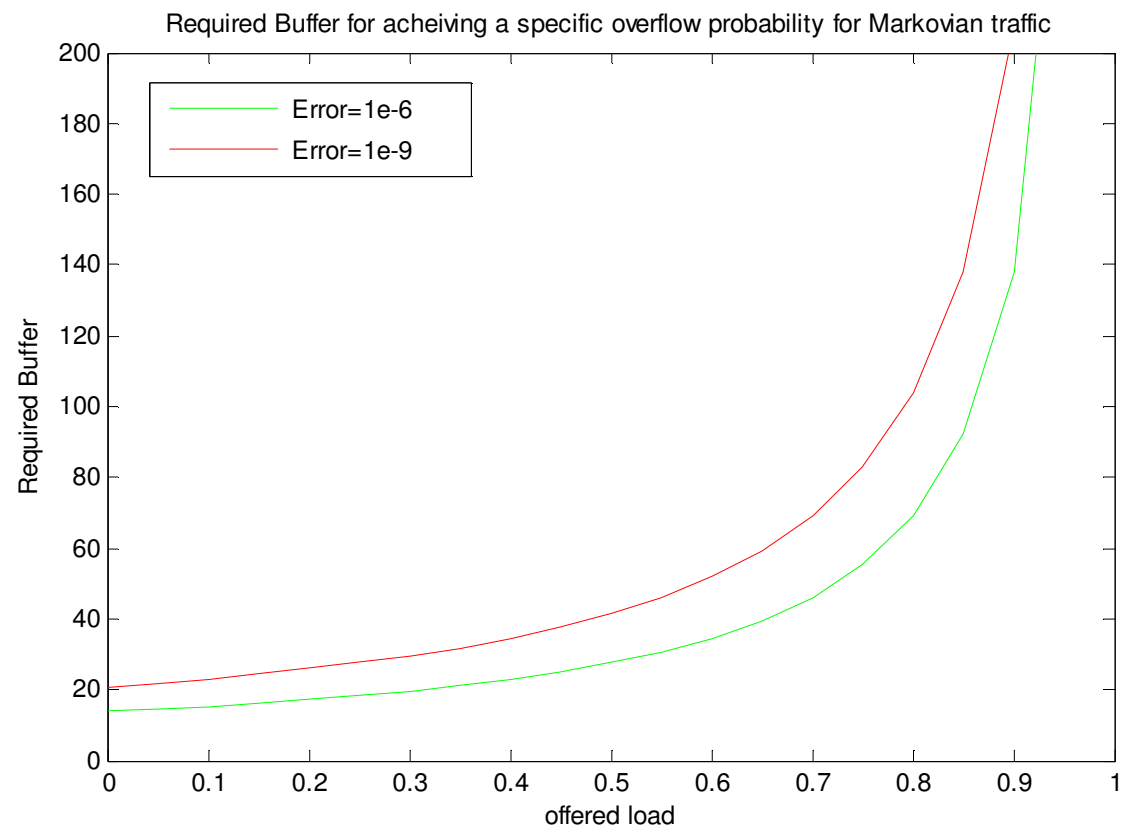

Figure 4. Required buffer vs offered load

\subsection{Performance Metric}

We use delivery ratio to measure the impact of varying buffer size on the performance metric. The delivery ratio is the ratio of total number of packets delivered at the client to the total number of packets generated at the source end. As shown in figure below, increasing buffer increases the delivery ratio.

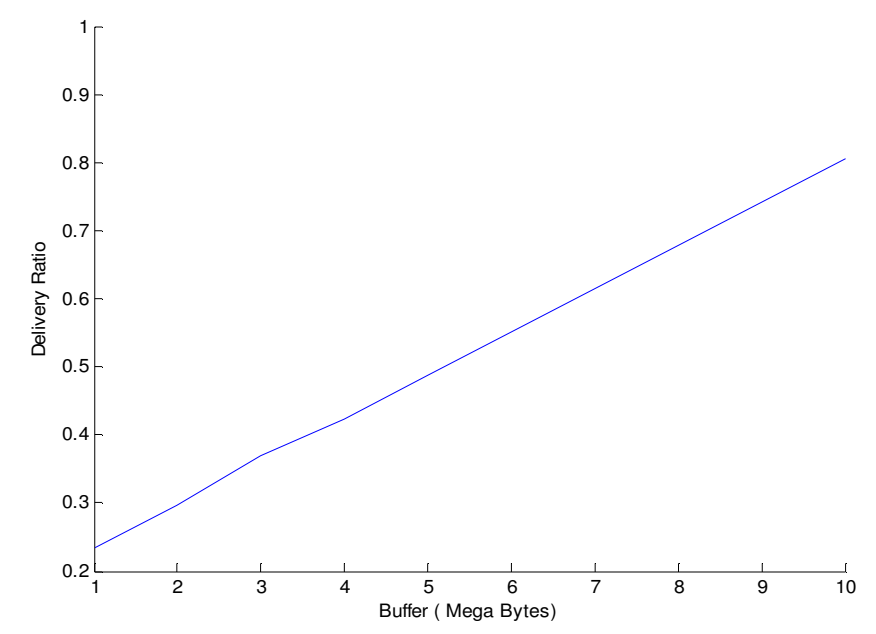

Figure 5. Impact of varying buffer size on Delivery ratio 
International Journal of Network Security \& Its Applications (IJNSA), Vol.6, No.4, July 2014

\section{END TO END DELAY CALCULATION AND BUFFER CONTROL}

The network could be modeled as a multihop clustered infrastructure where all nodes are connected with respect to dynamic control of end to end delay performance. The path connectivity between any pair of users is derived by using these nodes. When a source destination path is connected, the number of nodes in the path is random at each instance of path connection. The number of hops varies depending on the location of the source and destination. In addition, the delay variance at each node $\mathrm{n}$ a path is also random. The delay variability in each node is due to congestion and queuing delays. For a reasonable number of hops, this delay can be modelled as a Gaussian Process [2]. With each hop having a random process, the sum of processes that cause the delay can reach Gaussian. Thus end to end delay of IP packet transmission between the source and destination includes the delay variations caused by number of hops and variable delay within each node. Video source is encoded at $384 \mathrm{kbps}$ and each sample is represented by 8 bits. Therefore each sample is quantized using 256 levels at the transmission side. If we group every 256 byte of samples (2048 bits) into one packet, the corresponding Packet Generation Time (PGT) is 0.00533 seconds. We have chosen the uniform delay range for the multi hop connectivity between $70 \mathrm{~ms}$ and $130 \mathrm{~ms}$. In addition, each node has a time varying delay due to network traffic conditions based on a Gaussian process. This can be modeled with a nonstationary delay model based on using Gaussian process with a mean in the range of $[50,70] \mathrm{ms}$, and standard deviation in the range of $[50,100] \mathrm{ms}[2]$.

In summary the total delay in network is sum of PGT (Packet Generation Time), packet delay and network delay.

a) 5.33 ms Packet Generation Time

b) $5.33 \mathrm{~ms}$ Packetization Delay

c) A uniform distribution for random network delay

d) A Gaussian distribution for non-stationary network delay.

The PDF of the uniform distribution is

$$
f_{1}(x)=\frac{1}{130-70}, 70<x<130
$$

The PDF of the normal distribution is

$$
f_{2}(y)=\frac{1}{\sqrt{2 * \pi} \sigma_{2}} \exp \left(-\frac{\left(y-\mu_{2}\right)^{2}}{2 \sigma_{2}^{2}}\right)
$$

With mean $\mu_{2}$ and standard deviation $\sigma_{2}$

Using the above equations, it is possible to compute the average delay, variance of the delay and standard deviation of the delay.

The average delay is expressed as:

$$
\begin{aligned}
& \mathrm{E}(\text { delay })=\sum x_{i} p_{i}+\int x f_{1}(x) d x+\int y f_{2}(y) d y \\
& =10.66+\int_{-\infty}^{\infty} x f_{1}(x) d x+\int y f_{2}(y) d y
\end{aligned}
$$


International Journal of Network Security \& Its Applications (IJNSA), Vol.6, No.4, July 2014

$$
=10.66+\mu_{1}+\mu_{2}
$$

Since $\mathrm{X}$ and $\mathrm{Y}$ are independent, the variance of the delay is given by:

$\operatorname{Variance}=\operatorname{Var}(\mathrm{X})+\operatorname{Var}(\mathrm{Y})$

$$
=\sigma_{1}^{2}+\sigma_{2}^{2}
$$

Thus, the standard deviation of the delay is:

$$
\operatorname{Std}=\sqrt{\sigma_{1}^{2}+\sigma_{2}^{2}}
$$

The Coefficient of Variance (CV) which is used as the index for QoS control is given by:

$$
\mathrm{CV}=\frac{s t d_{n}}{\overline{t_{n}}}=\frac{\sqrt{\sigma_{1}^{2}+\sigma_{2}^{2}}}{10.66+\mu_{1}+\mu_{2}}
$$

Using the uniform distribution for multi-hop delay, we can compute the mean and the variance:

$\mu_{1}=100 \mathrm{~ms}$, variance

$\sigma_{11}^{2}=\left[(130-70)^{2}\right] / 12=300$

Using the Gaussian distribution for "end-to-end non-stationary" delay due to jitter, using the mean in the range $[50,70] \mathrm{ms}$ and the variance in the range $[50,100] \mathrm{ms}$, the $\mathrm{CV}$ for the end-toend path is given by:

Then $\mathrm{CV}=\frac{\sqrt{\sigma_{1}^{2}+\sigma_{2}^{2}}}{10.66+\mu_{1}+\mu_{2}}$

Here $\sigma_{1}=\sqrt{300}=17.3$ and $\mu_{1}=(130+70) / 2=100$

While if $\mathrm{CV}>0.33$, then we apply the control mode. In general, while $\mathrm{CV}>0.33$, let it be $\mathrm{Q}$, Then,

$$
\begin{aligned}
& \frac{\sqrt{\sigma_{1}^{2}+\sigma_{2}^{2}}}{10.33+\mu_{1}+\mu_{2}}=Q, \\
& \frac{\sqrt{300+\sigma_{2}^{2}}}{10.33+100+\mu_{2}}=Q
\end{aligned}
$$

Suppose for the control mode, we need to buffer the packets for $\mathrm{x} \mathrm{ms,} \mathrm{if} \mathrm{we} \mathrm{want} \mathrm{CV} \mathrm{to} \mathrm{reduce} \mathrm{to}$ 0.3 , we get 
$\frac{\sqrt{300+\sigma_{2}^{2}}}{10.66+100+\mu_{2}+x}=0.3$,

Here we take an example: suppose, $\mathrm{CV}=0.4$. Then we get

$\mathrm{CV}=\frac{\sqrt{\sigma_{1}^{2}+\sigma_{2}^{2}}}{64+\mu_{1}+\mu_{2}}=0.4$

Where,

$\mu_{1}=100 \mathrm{~ms}$, variance $\sigma_{1}=(130-70)^{2} / 12=\sqrt{300}$

Suppose for the buffer control mode, we need to buffer the packets for $\mathrm{x}$ ms, if we want CV to reduce to 0.3 , we get

$\mathrm{CV}=\frac{\sqrt{300+\sigma_{2}^{2}}}{64+100+\mu_{2}+x}=0.3$

Then we notice that $\left(64+100+\mu_{2}+x\right) * 0.3=\left(64+100+\mu_{2}\right) * 0.4$

If we know mean $\mu_{2}$, we can find the value of $\mathrm{x}$.

Based on the CV methodology, we maintain buffer not to overflow. We divide the network into two modes, namely normal and control mode based on $\mathrm{CV}$ value calculated using about equations. IF CV stays in the range of $0.27<\mathrm{CV}<0.33$ [2], we call it a normal mode and no action is necessary. If $\mathrm{CV}$ falls below or above these threshold range, network switches into control mode and actions are taken to bring $\mathrm{CV}$ to normal mode.

\section{CONCLUSION AND FUTURE WORKS}

Providing end to end QoS for video streaming applications is a key challenge in today's wireless mobile networks. The main finding of this paper is that buffer management can be used to optimize the performance of multimedia streaming over unreliable wireless links. In this Research, we studied the effect of receiver buffer size on the quality of perceived video streaming application. Our Objective was to keep receiver buffer as small as possible for a give interrupt free streaming. Our experiment shows how buffer size should be changed based on network variation. We built our simulation to determine the minimum buffer by increasing buffer size till there are no interrupts. We present performance for achieving the desired QoS for video streaming application by extending the use of tri mode control already used by Voice/IP application. The performance is based on using a finite buffer at the end user device, dynamically controlled by Coefficient of Variance.

We would like to extend this work in future by implementing theoretical model in real ad hoc environment network. We haven't considered mobility and channel impairment issues for which we need to deepen our study and investigate performance measure of our system in various mobility cases. We would also like to compare our algorithm with other existing bandwidth smoothing techniques used in video streaming. 
International Journal of Network Security \& Its Applications (IJNSA), Vol.6, No.4, July 2014

\section{REFERENCES}

[1] Dapeng Wu; Yiwei Thomas Hou; Ya-Qin Zhang, "Transporting real-time video over the Internet: challenges and approaches," Proceedings of the IEEE, vol.88, no.12, pp.1855,1877, Dec. 2000

[2] D. R. Vaman, S.T.Koay, Y. Zhang, T.Li, " Real Time Distributed Tri-Mode Control of Coefficient of Variance for High Quality of Service End-to-End Voice/IP Application", Proceedings of IEEE $20102^{\text {nd }}$ Conference on Future Computing and Communications (FCC 2010), Paper, 337, Shanghai, China, September 28-29, 2010.

[3] Bhadada, Sharma K L, "Evaluation and Analysis of buffer Requirements for streamed Video data in video on demand Application", IETE Journal of Research, Volume 56, 2010

[4] D.Wu, Y.T. Hou, W. Zhu, H.-J.Lee, T.Chiang and H.J.Chao, "On end to end architecture for transporting MPEG-4 video over Internet,” IEEE Trans. Circuits Syst. Video Technoloy, 10, pp 923-941, September 2000

[5] Dejian Ye; Qiufeng Wu; Zuo Zhang, "Receiver-buffer-driven approach to adaptive Internet video streaming," Electronics Letters, vol.38, no.22, pp.1405,1406, 24 Oct 2002.

[6] Jan, F.; Mathieu, B.; Meddour, D., "Video Streaming Experiment on Deployed ad hoc Network," Testbeds and Research Infrastructure for the Development of Networks and Communities, 2007. TridentCom 2007. 3rd International Conference on, vol., no., pp.1,10, 21-23 May 2007

[7] Dapeng Wu; Hou, Y.T.; Zhu, Wenwu; Ya-Qin Zhang; Peha, J.M., "Streaming video over the Internet: approaches and directions," Circuits and Systems for Video Technology, IEEE Transactions on , vol.11, no.3, pp.282,300, Mar 2001

[8] Kuroda, R.; Katsuki, M.; Otaka, A.; Miki, N., "Providing flow-based quality-of-service control in a large-scale network," Communications, 2003. APCC 2003. The 9th Asia-Pacific Conference on, vol.2, no., pp.740, 744 Vol.2, 21-24 Sept. 2003.

[9] Ng, J.K.-Y.; Shibin Song, "A video smoothing algorithm for transmitting MPEG video over limited bandwidth," Real-Time Computing Systems and Applications, 1997. Proceedings, Fourth International Workshop on, vol., no., pp.229,236, 27-29 Oct 1997.

[10] Lisha Gao and Junzhou Luo, "Performance Analysis of a P2P-Based VOIP Software", Proceedings of Telecommunications 2006, AICT/ICIW 2006, Advanced International Conference on Internet and Web Applications and Services, February 19-25, 2006, pp 11-14.

[11] D.R. Vaman, "Congestion Management through Buffering, Discarding and Scheduling Strategies”, Joint Megazess/NIST paper, Conference on Advanced Technologies, National Institution of Standards and Technology, July 20, 2000.

[12] Masugi, M.; Takuma, T.; Matsuda, M., "QoS assessment of video streams over IP networks based on monitoring transport and application layer processes at user clients," Communications, IEEE Proceedings- , vol.152, no.3, pp.335,341, 3 June 2005.

[13] Pereira, R.; Pereira, E.G., "Client Buffering Considerations for Video Streaming," Advanced Information Networking and Applications Workshops (WAINA), 2013 27th International Conference on , vol., no., pp.595,600, 25-28 March 2013.

[14] Kuroda, R.; Katsuki, M.; Otaka, A.; Miki, N., "Providing flow based quality of service control in a large scale network," Communications, 2003. APCC 2003. The $9^{\text {th }}$ Asia-Pacific Conference, vol.2, no., pp 740, 744 Vol. 2, 21-24 Sept.2003

[15] Yoshida, H.; Nogami, K.; Satoda, K., "Proposal and evaluation of Joint Rate Control for stored video streaming," Communications Quality and Reliability (CQR), 2010 IEEE International Workshop Technical Committee on, Vol., no., pp 1, 6, 8-10 June 2010.

[16] Chlamtac, M. Conti and J. J.-N. Liu, "Mobile ad hoc Networking: imperatives and challenges," Ad Hoc Networks, vol. 1, pp. 13-64 Elsevier, 2003

[17] H. Gharavi and K. Ban, "Dynamic packet control for video communications over ad-hoc networks". 2004 IEEE Int. Conf. on Communications, Paris, France, June 2004

[18] IETF, "MANET Working Group Charter," http://www.ietf.org/html. charters/manet-manet.html

[19] Qian Zhang, "Video Delivery over Wireless Multi-hop Networks," International Symposium on Intelligent Signal Processing and Communication Systems (ISPACS 2005), pp. 793-796, December 2005.

[20] S. Blake, D. Black, M. Carlson, E. Davies, Z. Wang, and W. Weiss, "An Architecture for Differentiated Services", RFC 2475, Oct 1998

[21] Aamir, Muhammad; Zaidi, Mustafa A, "A buffer management scheme for packet queues in MANET," Tsinghua Science and Technology , vol.18, no.6, pp.543,553, Dec. 2013 
International Journal of Network Security \& Its Applications (IJNSA), Vol.6, No.4, July 2014

[22] Zenghua Zhao; Shubing Long; Yantai Shu, "Rate Adaptive Live Video Streaming in Manets," Communications and Networking in China, 2006. ChinaCom '06. First International Conference on, vol., no., pp.1,5, 25-27 Oct. 2006.

[23] J. Broch, D. A. Maltz, D. B. Johnson, Y.-C. Hu, and J. Jetcheva, "A performance comparison of multi-hop wireless ad hoc network routing protocols," in Proc.ACM/IEEE Int. Conf. Mobile Computers and Networking, pp. 85-97, 1998.

[24] Hsiao-Chiang Chuang; ChingYao Huang; Tihao Chiang, "On the buffer dynamics of scalable video streaming over wireless network," Vehicular Technology Conference, 2004. VTC2004-Fall. 2004 IEEE 60th, vol.4, no., pp.2582,2586

[25] Ad hoc Networking, C.E. Perkins, Addison Wesley, Jan. 2001

\section{Authors}

Prabesh S Dongol received his Bachelor's degree in Engineering in Electrical and Communication Engineering from Kathmandu Engineering College, T.U, Nepal in 2005. He received his Masters of Science degree in Electrical Engineering from University of South Alabama, Mobile, AL, USA. Currently he is pursuing his Ph.D. degree in Electrical Engineering from Prairie View A\&M University, Prairie View, Texas. His research interests include multimedia networking, Quality of Support for multimedia over IP networks, wireless networks and satellite communications.

Dhadesugoor R. Vaman is Texas Instrument Endowed Chair Professor and Foun dng Director of ARO Center for Battlefield Communications (CeBCom) Research, ECE Department, Prairie View A\&M University (PVAMU). He has more than 38 years of research experience in telecommunications and networking area. He has over 25 years of experience in the telecommunications industry. Currently, he has been working on the control based mobile ad hoc and sensor networks with emphasis on achieving bandwidth

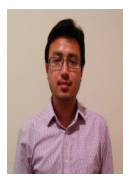
efficiency using KV transform coding; integrated power control, scheduling and routing in cluster based network architecture; QoS assurance for multi-service applications; and efficient network management. Dr. Vaman was previously a faculty advisor for NIST (the Department of Commerce) and has served as an industry advisor and consultant to GTE, Spacenet, Timeplex, Singer Kearfott, ITT, Inter Digital and Transwitch Corporations. He was also a professor in EECS, Stevens Institute of Technology, and the founding director of the Advanced Telecommunications Institute (ATI), U.S. Navy Center of Excellence in Telecommunications. He has won numerous awards and has published over 100 papers and co-authored a textbook. 